\title{
Caracterización de los procesos de obtención, transporte, procesamiento y criopreservación de las muestras de sangre de cordón umbilical, obtenidas de enero de 2006 a febrero de 2008 en el banco de células Stem de Colombia
}

\author{
Luz Mabel Ávila Portillo', Claudia Martínez², Jenniffer Ávila', \\ Andrea Becerra' ${ }^{1}$, Jesus Jaimes ${ }^{1,3}$, Claudia Gómez ${ }^{1}$, \\ José Ignacio Madero' \\ 1. Banco de Células Stem de Colombia. \\ 2. Estudiante Universidad Colegio Mayor de Cundinamarca, Bogotá, Colombia. \\ 3. Asesor Bioestadística externo. \\ Correspondencia: laboratorio@bancodecelulas.com \\ Recibido: 17-03-09 / Aceptado: 07-05-09
}

\section{Resumen}

La sangre de cordón umbilical ha pasado de ser un desecho biológico a ser una fuente potencial de células madre hematopoyéticas con capacidad regenerativa, por lo cual, el procesamiento y criopreservación después de su obtención en condiciones que garanticen su utilización es clave en un transplante exitoso. El almacenamiento de células madre hematopoyéticas de sangre de cordón umbilical es realizado tanto en bancos de carácter público como privado. Nuestro objetivo fue realizar un análisis de la experiencia de un banco de sangre de cordón umbilical en Colombia, en el cual se caracterizaron los procesos de obtención, transporte, procesamiento y criopreservación. Para ello se diseñó un estudio de serie de casos, en el que se analizaron 1080 registros de la base de datos del Banco de Células Stem de Colombia S.A. Este análisis evidenció que el tiempo transcurrido antes del procesamiento de las muestras fue menor de $12.7 \pm 10.3$ horas y el volumen promedio de 68,2 27,6 mL; el recuento inicial de células nucleadas tuvo un promedio de 1,03 × $109 \mathrm{~cm} /$

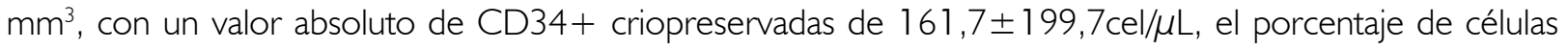
CD34 + de las muestras fue de $0,228 \%$, el $88,6 \%$ de las muestras estudiadas tenían un porcentaje de viabilidad superior al 95\%, 21 muestras que corresponden al 1,9\% presentaron hemocultivo positivo y el 100\% de las pruebas serológicas para enfermedades infectocontagiosas fueron negativas.

Palabras clave: células madre, cordón umbilical, criopreservación, leucemia mieloide, viabilidad celular.

\begin{abstract}
Characterization of the Processes of Obtaining, Transporting, Processing and Cryopreservation of Samples of Umbilical Cord Blood Obtained from January 2006 to February 2008 in Colombia Stem Cells Bank

Umbilical cord blood has gone from being a biological waste to being a potential source of hematopoietic stem cells with regenerative capacity, making, processing and cryopreservation after collection under conditions
\end{abstract}


that ensure their use is key to a successful transplant. The storage of hematopoietic stem cells from umbilical cord blood banks is conducted in both public and private character. Our objective was to analyze the experience of a bank of umbilical cord blood in Colombia, which characterizes the processes of production, transportation, processing and cryopreservation. To do this, we designed a case-series study, which analyzed 1080 records from the database of Stem Cell Bank of Colombia SA.

This analysis showed that the time elapsed before the processing of the samples was less than $12.7 \pm 10.3$ hours and the average volume from $68.2 \pm 27.6 \mathrm{~mL}$, the initial count of nucleated cells averaged $1.03 \times 109 \mathrm{~cm} / \mathrm{mm}^{3}$, with an absolute value of cryopreserved CD34 + of 161.7 \pm 199.7 cell / uL, the percentage of CD34 + cells from the samples was $0.228 \%, 88.6 \%$ of the studied samples had a viability rate higher than $95 \%, 2$ I samples corresponding to $1.9 \%$ had positive blood cultures and $100 \%$ of serological tests for infectious diseases were negative.

Key words: cell viability, cryopreservation, myeloid leukemia, stem cells, umbilical cord

\section{Introducción}

Las células madre hematopoyéticas $(\mathrm{CMH})$ provenientes de sangre de cordón umbilical (SCU) se han convertido en una alternativa terapéutica para muchas enfermedades, tales como Leucemia Mieloide Aguda (AML) (1), enfermedad de Krabbe (2) y síndrome de Wiskott Aldrich (3) entre otras; por lo cual los Bancos de Sangre de Cordón Umbilical (BSCU) desde sus inicios, en el año de 1993 en Nueva York, son cada vez más frecuentes $(4,5)$.

En Colombia existen tres bancos de criopreservación de SCU. Sin embargo, a pesar de la existencia de éstos, no hay hasta el momento una ley o norma que los rija en criterios como el transporte, recolección, procesamiento y criopreservación. Razón por la cual cada Banco de SCU ha definido sus criterios de acuerdo a su experiencia, que varía dependiendo del sitio de entrenamiento del personal técnico. Algunos se han entrenado en bancos de cordón públicos acreditados por NETCORD (6) y otros en Bancos de Unidades de Fertilidad. Por esta razón, se realizó un análisis de los procesos de recolección, de procesamiento y de criopreservación de muestras de SCU que fueron recolectadas y criopreservadas entre los meses de Enero de 2006 a Febrero del 2008 en el Banco de Células Stem de Colombia; banco privado con certificación ISO-9001 dirigido por profesionales entrenados en un banco público acreditado por NETCORD.

\section{Materiales y métodos}

\section{Obtención de datos}

Se realizó un estudio de serie de casos, descriptivo y retrospectivo de las muestras de SCU. Se analizaron 1080 registros de la base de datos del Banco de Células Stem de Colombia en un periodo comprendido entre Enero del 2006 a Febrero del 2008, estas muestras fueron remitidas de ciudades colombianas y del exterior. Sólo se tuvieron en cuenta las muestras de SCU que tenían como mínimo los datos de: volumen de SCU, recuento celular inicial y final, tiempo de procesamiento, número de células CD34+, \% de células CD34+, viabilidad, hemocultivos y pruebas de enfermedades infecciosas. La obtención y la revisión de la base de datos fueron autorizadas por el Banco de Células Stem de Colombia.

\section{Análisis estadístico}

Se elaboró una base de datos, que fue procesada con el paquete estadístico SPSS. ${ }^{\circledR}$ versión 11.05 para Windows ${ }^{\circledR}$. Se realizó un análisis descriptivo univariado, construyendo tablas y graficas, elaboradas con los estadígrafos según el nivel de medición de las variables, de manera que para las variables cualitativas se obtuvo la distribución porcentual de frecuencias mientras que para las variables cuantitativas se calcularon medidas de tendencia central. Posteriormente, se 


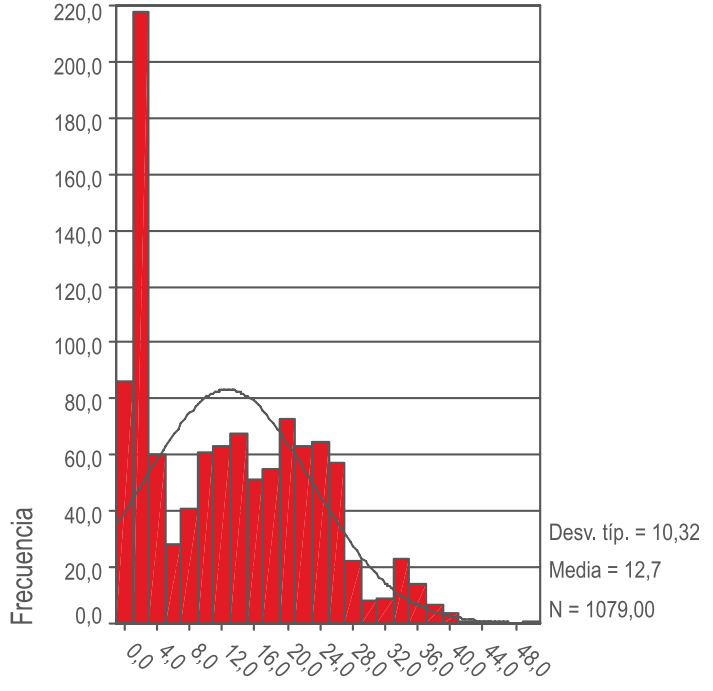

TIEMPO ANTES DEL PROCESAMIENTO
Figura 1. Distribución del tiempo de recolección de muestra antes del procesamiento. Desv. Típ: desviación típica. N: tamaño de la muestra.

hizo un análisis mediante la prueba de $\mathrm{Chi}^{2}\left(\chi^{2}\right)$ para valorar la fuerza de asociación. Es importante recalcar que para eliminar los efectos del control de sesgos y expectativas de los investigadores (validez), el procesamiento estadístico fue desarrollado por personal de bioestadística independiente del grupo clínico de investigación. Los datos obtenidos fueron comparados con los requisitos expuestos en la literatura mundial.

\section{Resultados}

Las muestras se tomaron durante procedimientos de cesárea $(73.3 \%)$ y de parto natural (26,7\%). Al realizar el análisis sobre el origen de las muestras procesadas, se pudo determinar que el $60.8 \%$ de éstas procedían de la ciudad de Bogotá, y el resto de otros lugares. Se hizo un análisis sobre el tiempo transcurrido entre la toma de la muestra y el inicio del procesamiento en el Banco de Células Stem de Colombia, encontrándose una media de $12.7 \pm 10.3$ horas, observándose una distribución multimodal asimétrica positiva con respecto a la media; indicando que en la mayoría de las muestras, el tiempo de transporte fue menor de $12.7 \pm 10.3$ horas, Figura 1 .

\section{Caracterización del procesamiento}

Se realizó una caracterización del procesamiento y obtención de células. El volumen de SCU presentó un promedio de $68,2 \pm 27,6 \mathrm{~mL}$, con un valor máximo de
VOLUMEN DE LA MUESTRA

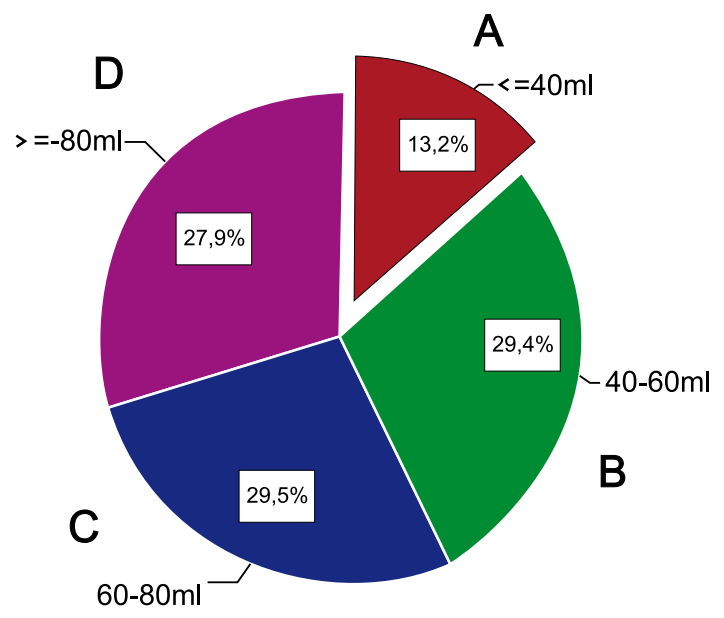

Figura 2. Categorización del volumen de la muestra.

$240 \mathrm{~mL}$. Para una mejor evaluación se categorizaron en los niveles A: <40 mL, B: 40-60 mL, C: 60-80 mL y D: >80 $\mathrm{mL}$. En la Figura 2 se muestra los porcentajes obtenidos en cada uno de las categorías.

El recuento inicial de células nucleadas presentó un promedio de $103.0 \times 107$, con un mínimo de 117.0 1 x06 y un máximo de 453.0 x107. Se encontró que las células totales post procesamiento fueron de 581 x 106 en promedio. A partir de lo anterior se pudo analizar que en el procedimiento de reducción de volumen se perdieron en promedio 455.0x106 células mononucleares (CMN) oscilando entre un mínimo de 246x104 y un máximo de $356 \times 107$, con una perdida en general del 30\%.

\section{Células progenitoras hematopoyeticas (CD34+)}

En cuanto al número de células CD34+, se observa en la Tabla 1 que el promedio absoluto fue $161,7 \pm 199,7 \mathrm{cel} / \mu \mathrm{L}$, con un porcentaje promedio de $0,228 \%$, que osciló entre un mínimo $0,06 \%$ y un máximo de $4,79 \%$.

Se procedió a categorizar el porcentaje de CD34+ obtenido utilizando los criterios recomendados por la literatura $(6,7)$ que se observa en la Figura 3, donde el $70.7 \%$ de las muestras tiene un porcentaje superior al $0.1 \%$ de CD34+.

Respecto a la viabilidad, se puede afirmar que en el $88,6 \%$ de las muestras tuvo un porcentaje superior al $95 \%$. Finalmente, se analizaron los resultados de pruebas infecciosas 
Tabla1. Datos descriptivos de células CD34+.

\begin{tabular}{ccccccc} 
& $\mathbf{N}$ & Media & Mediana & ET & Mín & Máx \\
\hline CD34/ul & 1080 & 161,7 & 78.76 & 199,65 & 4.09 & 1352 \\
TOTAL CD34 & 1080 & $3.234 .086,11$ & 1.575 .200 & $3.993 .194,16$ & 81.800 & 27.020 .000 \\
\%CD34 & 1079 & 0.22 & 0,15 & .292409 & .006 & 4.790 \\
\%VIAB & 1079 & 98,12 & 99,36 & 304.244 & 80.6 & 100 \\
\hline
\end{tabular}

ET: Error típico, Min: valor mínimo, Máx: Valor Máximo, VIAB: Viabilidad

$\% \mathrm{CD} 34$

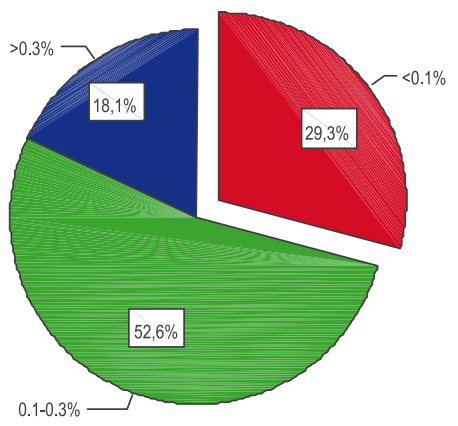

Figura 3. Distribución por categorías del porcentaje de CD34+ de la muestra.

(VIH1-2, VHB, VHC, VDRL) y de contaminación (hemocultivo), encontrándose que $21(1,9 \%$ ) presentaron hemocultivo positivo, todas ellas tomadas durante el parto natural (las cuales fueron informadas y descartadas inmediatamente). Los resultados para pruebas infecciosas fueron negativas en el 100\% de los casos.

\section{Análisis de asociaciones}

$\mathrm{Al}$ evaluar la relación entre el volumen de la muestra y el recuento de células totales iníciales se encontró una asociación significativa con base en la prueba de Chi cuadrado con $\mathrm{p}<0.05$, como se puede ver en la Figura 4; donde la proporción de células iníciales $<4 \times 108$ disminuye a medida que se incrementa el volumen, generando una mejor calidad de las muestras almacenadas. En la Figura 5 se muestra el porcentaje de CD34+, que presentó una asociación significativa con valor $\mathrm{p}<0.001$. La obtención de células CD34+ es directamente proporcional al volumen de la muestra.

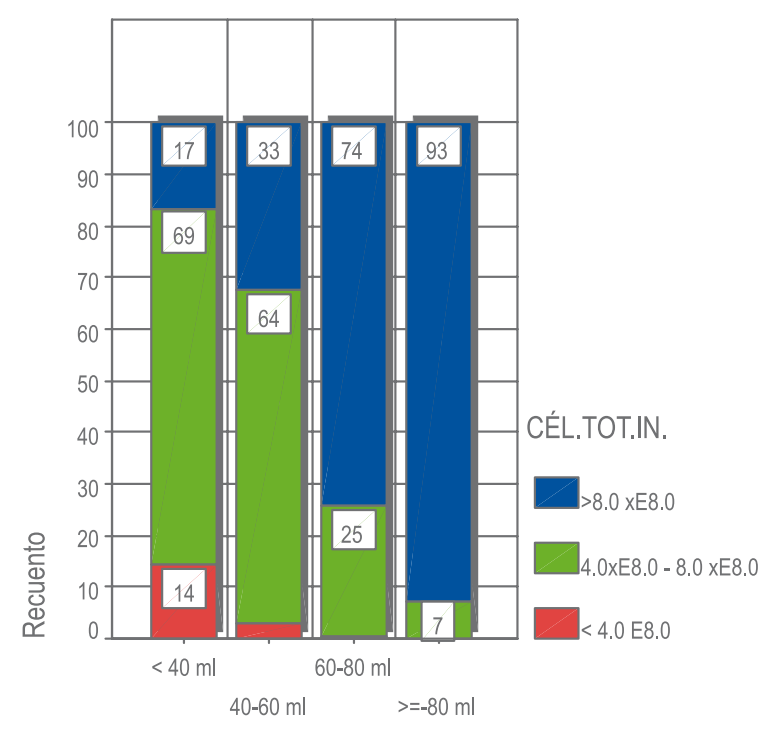

Figura 4.Volumen en correlación con el recuento celular. CÉL. TOT.IN: Células totales iniciales; los cuadros grandes encierran tamaño de la muestra para cada clasificación.

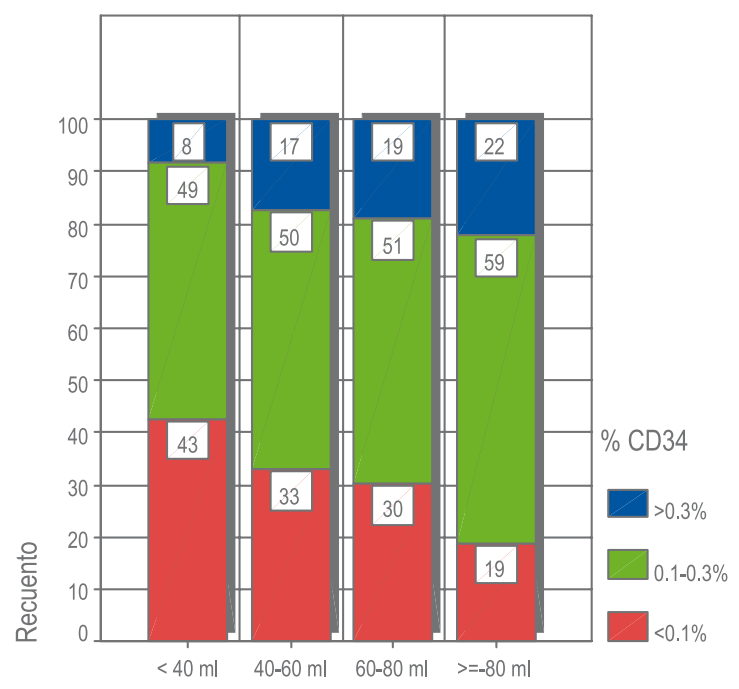

Figura 5. Porcentaje de CD34 en relación al volumen de la muestra. Los cuadros blancos encierran el tamaño de la muestra para cada asociación. 


\section{Discusión}

El protocolo de recolección y procesamiento de las muestras de SCU para almacenamiento de $\mathrm{CMH}$ en el Banco de Células Stem de Colombia es basado en el protocolo básico publicado por Rubinstein en 1995 (7), que consiste en un proceso manual de separación bajo un sistema cerrado estéril. La crío-preservación es realizada utilizando DMSO como crío-preservante y el proceso de congelamiento es efectuado mediante una curva de enfriamiento controlada en un equipo automatizado, y finalmente son almacenadas en nitrógeno líquido a $-196^{\circ} \mathrm{C}$ (8). El número de CMH CD34+ y la viabilidad es verificado por el conteo de células CD34 por técnica de citometría de flujo.

Para determinar la eficiencia de este sistema, se estableció que el tiempo transcurrido desde la toma de la muestra y el procesamiento fue de $12.7 \pm 10.3$ horas, siendo este tiempo óptimo para mantener la calidad de la muestra, ya que como se mostró en un estudio reportado por el Banco de Células Stem de Colombia, la viabilidad disminuye un $30 \%$ después de las 48 horas de la toma de la muestra (9), encontrándose dentro del parámetro de tiempo sugerido por la norma NETCORD FACT (10).

La reducción de volumen manual utilizando una bolsa Transfer Freezing bag ${ }^{\oplus}$ con sistema cerrado, genera una disminución del 30\% de la recuperación de la muestra. Datos similares fueron reportados en comunicación personal con instituciones que procesan manualmente. Mientras CordMx (Banco de Sangre de Cordón Mexicano), quien procesa bajo un sistema de separación y concentración celular automatizado (Sepax-Biosafe), reportó, en el 2005, un porcentaje de recuperación de un $80 \%$ (11), disminuyendo la pérdida celular, por lo cual este sistema es recomendado por la red internacional NETCORD FACT (6), que el Banco de Células Stem implementará en los próximos años.

Los criterios de aceptación utilizados en el Banco de Células Stem de Colombia, entre los que se encuentra un volumen mínimo de $40 \mathrm{~mL}$, también fueron analizados, encontrándose un volumen de muestra promedio de $68,2 \pm 27,6 \mathrm{~mL}$ con un máximo de $240 \mathrm{~mL}$. Al realizar una clasificación de las muestras se pudo determinar que un $13.2 \%$ se encuentran con un volumen bajo y un $29.5 \%$ de las muestras recibidas tenían un volumen superior o igual a $80 \mathrm{~mL}$, consideradas desde este aspecto, como muestras óptimas. Es importante anotar que el $56.4 \%$ superaban los $60 \mathrm{~mL}$, criterio considerado como deseable para su posterior utilidad.

CordMx maneja un volumen mínimo para procesamiento de $80 \mathrm{~mL}$ (11). Hay que tener en cuenta que el contraste de volumen está directamente relacionado con la posibilidad de realizar el congelamiento de las muestras con bajo volumen, con la aprobación de los padres, previa información de las posibilidades de utilización y la firma del consentimiento informado en los bancos privados, mientras que en los públicos, la muestra es regida por los criterios y las decisiones exclusivamente del Banco.

El segundo criterio de aceptación de una muestra de SCU es el recuento inicial de células nucleadas, siendo este de 400 millones. Se encontró un promedio de $103.0 \mathrm{x}$ $107 \mathrm{cmn} / \mathrm{mm} 3$ teniendo como valores mínimos y máximos 117.0 x106 y 453.0 x107 respectivamente. El número de CD34+ criopreservadas en el Banco de Células Stem de Colombia (cuyo análisis se realiza en la plataforma de citometría de flujo estandarizada por la sociedad internacional de ingeniería de tejidos hematopoyéticos ISHAGE) (12) arrojó un promedio de 3.23 x 104 similar a los reportados por CordMx de 2.68 x106 (11).

Se puede decir que los parámetros analizados en el banco de Células Stem de Colombia, manejan una media similar a los regidos por NETCORD, pudiendo así garantizar los productos criopreservados y las técnicas realizadas en el laboratorio para el procesamiento y almacenamiento de células Stem hematopoyéticas de SCU. NOV

\section{Referencias}

1. Takahashi S, Unrelated Cord Blood transplantation for adult patients with de novo acute myeloid leukemia. Blood 2004;103:489-91.

2. Escolar ML, Poe MD, Provenzale JM, Richards KC, Allison J, Wood $\mathrm{S}$, et al. Transplantation of umbilical-cord blood in babies with infantile Krabbe's disease. N Engl J Med; 2005 352:2069-2081.

3. Slatter MA, Gennery AR. Umbilical cord Stem cell transplantation for primary immunodeficiencies. Expert Opin Biol Ther 2006;6:555-565.

4. Rubinstein P. Adamson JW, Stevens C. The Placental/Umbilical Cord Blood Program of the New York Blood Center A Progress Report. Ann N Y Acad Sci. 1999;872:328-335.

5. García J, Amat LI, Querol S. Los Bancos de Sangre de Cordón Umbilical: una nueva contribución al tratamiento de las enfermedades hematológicas. Asociación Española de Científicos. [serial online] [6 pantallas] https://www.serina.es/escaparate/verpagina. cgi?idpagina $=1499 \&$ refcompra $=$ 
6. Net Cord Virtual Oficce. Home page. https://www.netcord.org/index. html. [On line]. [14.08. 08].

7. Rubinstein P, Dobrila L, Rosenfield RE. Processing and cryopreservation of placental/umbilical cord blood for unrelated bone marrow reconstitution. Proc Natl Acad Sci USA. 1995;92:10119-10122.

8. Ávila LM, Madero JI, López C, León MF, Acosta L, Gómez C, et al. Fundamentos de criopreservación. Rev Colomb Obstet Ginecol. 2006; 57:291-300.

9. Avila LM, Becerra A, Jaimes D, Guzmán J, Ávila JP, Madero-Cervera J. Evaluation of the viability and functionality of the progenitor blood cells from the umbilical cord thawed in a Stem cell Bank in Colombia. 2009. Art in press.
10. NetCord-FACT. International Standards for Cord Blood Collection, Processing, Testing, Banking, Selection, and Release. [serial online] December 2006. [citado el 10 feb 2008]; Third Edition [85 pantallas] http://www.ims.u-tokyo.ac.jp/Cell_Processing_and_Transfusion/ Netcord-FACT\%20standard3rd.pdf.

11. Calderón-Garcidueñas ED. Evaluación del programa nacional de sangre placentaria CordMX. Logros y expectativas. Rev Med Inst Mex Seguro Soc 2005;43:127-129 Supl 1.

12. Sutherland DR, Anderson L, Keeney M, Nayar R, Chin-Yee I. The ISHAGE guidelines for $\mathrm{CD} 34+$ cell determination by flow cytometry. International Society of Hematotherapy and Graft Engineering. J. Hematother. 1996; 5:213-226. 